\title{
ESTUDO ELETROANALÍTICO DO HERBICIDA PARAQUAT EM SOLUÇÕES AQUOSAS POR VOLTAMETRIA DE ONDA QUADRADA UTILIZANDO ULTRAMICROELETRODOS
}

\author{
Djenaine de Souza e Sergio A. S. Machado* \\ Instituto de Química de São Carlos, Universidade de São Paulo, CP 780, 13560-970 São Carlos - SP
}

Recebido em 18/7/02; aceito em 21/2/03

\begin{abstract}
ELECTROANALYTICAL STUDY OF THE PARAQUAT HERBICIDE IN AQUEOUS SOLUTION BY SQUARE WAVE VOLTAMMETRY USING ULTRAMICROELECTRODES. The electrochemical behavior of paraquat on Pt, Au and carbon fiber ultramicroelectrodes were studied in laboratory samples by square wave voltammetry at high frequencies. The results showed two reversible peaks for paraquat reduction, in agreement to the literature data. The first peak was associated to the reduction of paraquat molecule in solution, with the further adsorption of the intermediate on the electrode surface. This adsorbed species undergoes to electroreduction in a reaction associated to the second voltammetric peak. The variation in $\mathrm{pH}$ and square wave parameters showed the best conditions to reduce paraquat as $\mathrm{pH} 5.0$, frequency as high as $1000 \mathrm{~s}^{-1}$, scan increment of $2 \mathrm{mV}$ and square wave amplitude of $50 \mathrm{mV}$. At such conditions, a variation of paraquat concentrations from $4.3 \times 10^{-6}$ to $1.66 \times 10^{-4} \mathrm{~mol} \mathrm{~L}^{-1}$ presented values for the detection limit equal to 3.9, 6.2 and $20.3 \mathrm{ppb}$ on $\mathrm{Pt}$, Au and carbon, respectively, at $1000 \mathrm{~s}^{-1}$. These values are quite below ${ }^{17}$ the allowed limit of paraquat in drinking water.
\end{abstract}

Keywords: square wave voltammetry; paraquat; ultramicroelectrodes.

\section{INTRODUÇÃO}

O 1,1'-dimetil-4,4'-bipiridina-dicloreto foi introduzido na década de 30 , porém somente começou a ser utilizado como indicador redox na década de 50. Ele é comumente chamado de metil-viologeno por, na forma reduzida, formar um composto de cor azul ou violeta. Suas propriedades como pesticida foram avaliadas em 1958 e alguns anos depois foi introduzido comercialmente com o nome de paraquat ${ }^{1}$. Na Figura 1 é apresentada a sua fórmula estrutural.

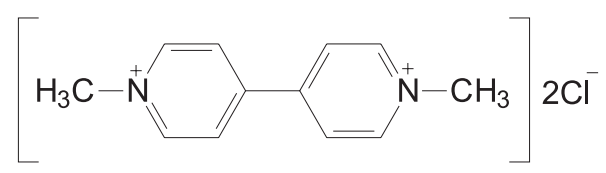

Figura 1. Fórmula estrutural do pesticida paraquat

$\mathrm{Na}$ forma de um sal de cloreto, ele é um herbicida não seletivo largamente utilizado e conhecido comercialmente como Gramoxone ${ }^{\circledR}$, Weedol ${ }^{\circledR}$ ou Panthclear ${ }^{\circledR}$.

O paraquat é extremamente tóxico, podendo causar intoxicações fatais em humanos e animais. No homem, ele pode ser reduzido pela enzima NADPH (fosfato de nicotinamida adenina dinucleotídeo)citocromo $\mathrm{P} 450$ redutase, com a transferência de um elétron, formando o radical paraquat ${ }^{2}$. Este, por sua vez, em presença de oxigênio oxida-se rapidamente produzindo um ânion radical superóxido e regenerando o paraquat. Desta maneira, ciclos repetidos de redução e re-oxidação do herbicida podem ocorrer gerando uma grande quantidade de espécies de oxigênio reduzido que levam o organismo ao stress oxidativo ou à peroxidação de gorduras.

Ele tem sido determinado por diversas técnicas analíticas como espectrofotometria ${ }^{3}$, análise em fluxo ${ }^{4}$, cromatografia líquida com detector de massa ${ }^{5}$, polarografia ${ }^{6}$ e imunoensaios ${ }^{7}$.

*e-mail: sasmach@iqsc.usp.br
Eletroquimicamente, tem sido investigado em várias superfícies eletródicas, incluindo eletrodos sólidos ${ }^{8}$, eletrodos de mercúrio ${ }^{6} \mathrm{e}$ eletrodos quimicamente modificados ${ }^{9}$. Contudo, o uso de ultramicroeletrodos aliado à voltametria de onda quadrada até o momento não havia sido mencionado em trabalhos da área.

A voltametria de onda quadrada (SWV) é uma técnica de pulso onde o pico de corrente resultante é proveniente da aplicação de pulsos de potenciais de altura $\Delta \mathrm{E}_{\mathrm{p}}$ (amplitude), que variam de acordo com uma programação de potenciais em forma de escada, com altura $\Delta \mathrm{E}_{\mathrm{s}}$ (incremento) e duração $2 \mathrm{t}$ (período). As correntes elétricas são medidas de forma diferencial ao final dos pulsos diretos e reversos e o sinal é dado como sendo a intensidade da corrente resultante. Por isto, o método apresenta excelente sensibilidade e alta rejeição às correntes capacitivas ${ }^{10}$.

As curvas de corrente-potencial apresentam perfil bem definido e são, geralmente, simétricas porque as correntes são medidas somente no final de cada semiperíodo e as variações na altura e na largura do pulso de potencial são sempre constantes, para um determinado intervalo de potenciais ${ }^{11}$.

Do ponto de vista prático, a maior vantagem da SWV é a facilidade na apresentação de um pico simétrico com maior intensidade de corrente que os picos obtidos na voltametria cíclica, e a facilidade em medir as correntes faradaicas num intervalo de tempo onde as correntes capacitivas são negligenciáveis. Isto se deve ao fato de que na SWV, assim como em outras técnicas de pulso, a corrente capacitiva diminui mais rapidamente que a corrente faradaica, de maneira que as medidas de corrente são realizadas após a corrente capacitiva ter se minimizado, melhorando significativamente a sensibilidade da técnica.

O desenvolvimento da parte eletrônica da instrumentação utilizada em eletroanalítica, principalmente nas medidas de correntes muito pequenas, e o surgimento de materiais com estruturas micrométricas proporcionaram as ferramentas necessárias que possibilitaram o uso de ultramicroeletrodos em técnicas modernas de pulso.

As propriedades relacionadas às dimensões dos ultramicroeletrodos, como as baixas correntes que circulam pela célula, permi- 
tem seu uso no estudo de reações e processos eletródicos em solventes de baixa condutividade e na ausência de eletrólito de suporte, ampliando os limites da investigação de processos eletroquímicos. O estudo de processos de transferência eletrônica rápida de elétrons e reações químicas acopladas também é facilitado pelo uso de ultramicroeletrodos ${ }^{12}$.

Os ultramicroeletrodos podem ser utilizados em várias técnicas eletroquímicas tradicionais, como voltametria cíclica, de varredura linear e cronoamperometria, como nas técnicas mais recentes, como voltametria de onda quadrada, voltametria de pulso normal e diferencial.

A utilização de ultramicroeletrodos promove um aumento intenso na qualidade dos dados experimentais. Estes incluem aumento da resolução, aumento na densidade de corrente e diminuição nos efeitos da resistência da solução ${ }^{13}$.

De acordo com as considerações acima, o objetivo deste trabalho foi estudar o comportamento eletroanalítico do herbicida paraquat em meio aquoso, utilizando diferentes ultramicroeletrodos aliados à técnica de voltametria de onda quadrada. As variações dos parâmetros experimentais e voltamétricos foram realizadas, com o intuito de se avaliar as condições em que é possível se obter as melhores respostas voltamétricas. Desta forma, espera-se otimizar os valores de limite de detecção e conseqüentemente, em trabalhos futuros, aplicar uma metodologia eletroanalítica para a determinação de paraquat em amostras de águas contaminadas.

\section{PARTE EXPERIMENTAL}

Os eletrodos de trabalho utilizados foram ultramicroeletrodos construídos no próprio laboratório utilizando-se microfios de platina com $25 \mu \mathrm{m}$ de diâmetro, microfios de ouro com $25 \mu \mathrm{m}$ diâmetro, e fibra de carbono com $7 \mu \mathrm{m}$ de diâmetro. Para a construção dos ultramicroeletrodos de platina e ouro, os microfios com $3 \mathrm{~cm}$ de comprimento foram soldados em um fio de cobre com $5 \mathrm{~cm}$ de comprimento e cerca de $0,5 \mathrm{~mm}$ de espessura, utilizando-se solda de ponto. Após a soldagem, os microfios foram inseridos em capilar de vidro Pyrex ${ }^{\circledR}$ e posteriormente preenchidos com resina epoxi. Como eletrodo de referência foi utilizado um eletrodo de $\mathrm{Ag} / \mathrm{AgCl}$ em $\mathrm{KCl}$ $3 \mathrm{~mol} \mathrm{~L}{ }^{-1}$, também construído no próprio laboratório.

Todos os experimentos eletroquímicos foram realizados em um Potenciostato/Galvanostato modelo 283 da EG\&G PARC, acoplado a um microcomputador Pentium $200 \mathrm{MHz}$, dotado de uma interface do tipo GPIB que utiliza o programa M 270 também da EG\&G PARC para coleta dos dados experimentais.

Em todas as análises os reagentes utilizados são de grau analítico, e o paraquat utilizado foi obtido comercialmente da Merck com pureza de $99,8 \%$

\section{RESULTADOS E DISCUSSÃO}

\section{Testes de eletroatividade}

A eletroatividade do paraquat foi testada sobre as superfícies dos UMEs de Pt, Au e fibra de carbono nos diferentes eletrólitos suporte: $\mathrm{Na}_{2} \mathrm{SO}_{4}, \mathrm{NaClO}_{4}$, e $\mathrm{KCl}$, todos na concentração de $0,1 \mathrm{~mol} \mathrm{~L}^{-1}$ utilizando a técnica de voltametria cíclica. Observou-se, para as varreduras de potenciais efetuadas entre $0 \mathrm{~V}$ e $-1,5 \mathrm{~V}$, a presença de dois picos de redução, próximos a $-0,65 \mathrm{~V}$ e $-0,98 \mathrm{~V}$, para os três UMEs utilizados, sendo que esta resposta foi mais intensa em $\mathrm{Na}_{2} \mathrm{SO}_{4}$ $0,1 \mathrm{~mol} \mathrm{~L}^{-1}$.

Para os três UMEs utilizados, nos diferentes eletrólitos de suporte em que foram realizados os testes, observou-se que praticamente não ocorreu deslocamento dos valores dos potenciais de pico, indicando que o processo eletroquímico é praticamente independente da natureza das superfícies dos UMEs. Este comportamento é indicativo de que o processo de adsorção do intermediário formado na primeira reação (pico 1) é bastante forte, independendo da natureza da superfície eletródica.

\section{Otimização das condições experimentais}

Definido o intervalo de potenciais a ser explorado no estudo do comportamento eletroanalítico do paraquat, foi feita uma otimização de parâmetros utilizando a voltametria de onda quadrada em $\mathrm{Na}_{2} \mathrm{SO}_{4}$ $0,1 \mathrm{~mol} \mathrm{~L}^{-1}$.

A variação da concentração hidrogeniônica do meio foi realizada acrescentando-se ao eletrólito, de $\mathrm{Na}_{2} \mathrm{SO}_{4} 0,1 \mathrm{~mol} \mathrm{~L}^{-1}$, quantidades suficientes de uma solução estoque, $0,1 \mathrm{~mol} \mathrm{~L}^{-1}$, de $\mathrm{H}_{2} \mathrm{SO}_{4}$ ou $\mathrm{NaOH}$.

Para os três UMEs utilizados, o aumento do $\mathrm{pH}$ do meio, a partir de um valor igual a 2 , provocou um ligeiro aumento na intensidade das correntes de pico até pH 5, após este valor a intensidade de corrente voltou a diminuir para os dois picos de redução. Este valor de $\mathrm{pH}$ associado a um máximo de corrente geralmente está relacionado ao valor de $\mathrm{pK}_{\mathrm{a}}$ da molécula eletroativa.

Desta maneira, para fins analíticos o valor de $\mathrm{pH}$ utilizado para o desenvolvimento da metodologia de análise do herbicida paraquat foi 5,0, que corresponde a uma solução de $\mathrm{Na}_{2} \mathrm{SO}_{4} 0,1$ mol L-1.

A separação das correntes direta, reversa e resultante na SWV possibilitou uma observação preliminar sobre o tipo de processo redox que ocorre na molécula do pesticida no meio em estudo. A presença de um pico de corrente no sentido reverso da varredura pode ser o indicativo da ocorrência de um processo reversível ou quase-reversível. A Figura 2 apresenta os voltamogramas com as respectivas separações das componentes de corrente direta, reversa e resultante para os UMEs de Pt, Au e FC, respectivamente. Nestes voltamogramas pode-se observar a presença de dois picos na varredura de potenciais no sentido direto e os correspondentes dois picos no sentido reverso, em potenciais idênticos àqueles obtidos anteriormente com a voltametria cíclica.

A frequiência de aplicação dos pulsos de potenciais é uma das mais importantes ferramentas da SWV, pois o seu aumento melhora significativamente as respostas analíticas e, além disso, fornece importantes informações acerca do comportamento redox da espécie de interesse. Uma avaliação do comportamento da corrente de pico em função da

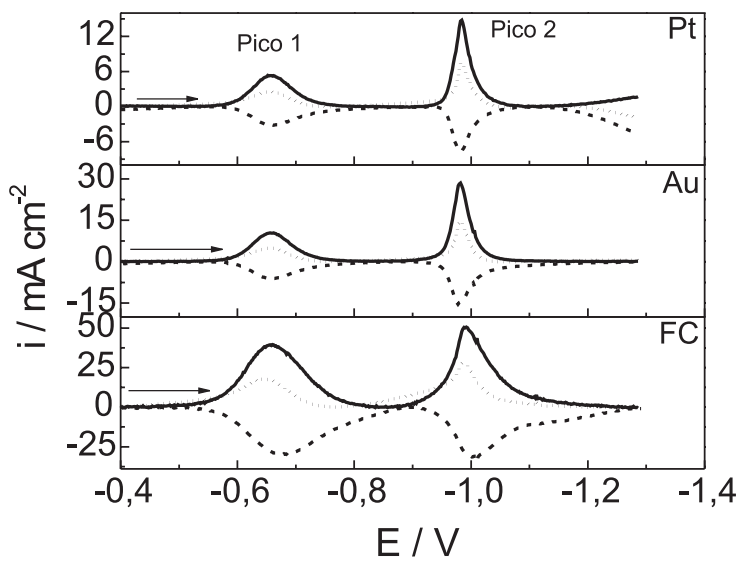

Figura 2. Componentes de corrente para $2,1 \times 10^{-5}$ mol $L^{-1}$ de paraquat em meio de $\mathrm{Na}_{2} \mathrm{SO}_{4} 0,1 \mathrm{~mol} \mathrm{~L}^{-1}$ em UME de Pt, Au e FC, com $\Delta E_{p}=50 \mathrm{mV}$, $\Delta E_{s}=2 m V$ e $f=500 \mathrm{~s}^{-1}$, onde (---) corrente direta, (....) corrente reversa e (-) corrente resultante 
frequiência de aplicação dos pulsos de potencial, para os três UMEs, mostrou que os dois picos se comportam de maneira diferente.

Para o pico 1, a corrente é diretamente proporcional à raiz quadrada da frequiência de aplicação de pulsos de potencial $\left(I_{p} \propto f^{1 / 2}\right)$. Para o pico 2, a corrente de pico é diretamente proporcional à freqüência de aplicação dos pulsos de potencial $(I \propto f)$. Seguindo os critérios de diagnósticos desenvolvidos para a SWV ${ }^{10,11}$, estes comportamentos correspondem ao controle da reação por difusão e por adsorção de produto e/ou reagente, respectivamente. Estes dados estão de acordo com a literatura ${ }^{8}$, onde se encontra que o produto da redução formado na primeira etapa (pico 1) se adsorve à superfície do eletrodo, reduzindo-se na segunda etapa (pico 2).

Este crescimento contínuo da corrente de pico com o aumento da freqüência de aplicação dos pulsos de potencial mostrou que é muito vantajosa, do ponto de vista analítico, a escolha das freqüências mais elevadas para o trabalho de rotina. Com isto, selecionou-se a freqüência de $1000 \mathrm{~s}^{-1}$ para os estudos de SWV, objetivando-se determinar uma curva analítica para o paraquat em águas naturais, para os três UMEs utilizados.

Esta possibilidade representa um avanço na determinação eletroanalítica deste importante pesticida em eletrólitos aquosos, pois o emprego de altas frequiências nesta análise encontra alguns fatores limitantes. Por exemplo, Zen ${ }^{14}$ e colaboradores utilizaram eletrodos modificados com Nafion ${ }^{\circledR}$ e observaram, para valores de frequiências superiores a $90 \mathrm{~s}^{-1}$, o surgimento de uma corrente residual muito elevada, o que dificultava as análises. Walcarius e Lamberts ${ }^{6}$ empregaram o HMDE para analisar o paraquat. Neste caso, encontrou-se um limite superior de frequiências de $200 \mathrm{~s}^{-1}$, que os autores deixaram de analisar com profundidade, associando-o ao aumento da corrente capacitiva. Neste trabalho, o uso de UMEs permite superar este tipo de limitação, devido a suas características como, por exemplo, a baixa sensibilidade a correntes capacitivas, o que resulta em significativa melhora na sensibilidade. Esta é uma das vantagens inerentes ao uso dos UMEs, ampliando a janela de parâmetros experimentais acessíveis em experimentos eletroanalíticos e melhorando consideravelmente a sensibilidade das técnicas voltamétricas.

A amplitude de pulso de potenciais e o incremento de varredura também foram avaliados a fim de se observar os valores em que melhores respostas em termos de intensidade de corrente e perfil voltamétrico fossem obtidas. Desta maneira, os valores otimizados para todos os parâmetros são apresentados na Tabela 1.

Tabela 1. Parâmetros otimizados para a detecção do paraquat em UME de Pt, Au e FC

\begin{tabular}{lc}
\hline Parâmetros Voltamétricos & Condições Otimizadas \\
\hline Incremento de varredura $\left(\Delta \mathrm{E}_{\mathrm{s}}\right)$ & $2 \mathrm{mV}$ \\
Amplitude de onda quadrada $\left(\Delta \mathrm{E}_{\mathrm{p}}\right)$ & $50 \mathrm{mV}$ \\
Freqüência de onda quadrada $(f)$ & $1000 \mathrm{~s}^{-1}$ \\
pH do eletrólito de suporte & 5,1 \\
\hline
\end{tabular}

Para os UMEs de Pt e Au e FC, o tempo e o potencial de condicionamento não tiveram nenhuma influência nas correntes e potenciais de pico, mostrando que não ocorreu nenhum acúmulo da espécie redox na superfície do UME. Com isto, para fins analíticos, a aplicação de potenciais de condicionamento não melhorou as respostas voltamétricas para nenhum dos UMEs em nenhum dos potenciais de acúmulo avaliados.

\section{Curvas analíticas para o paraquat}

Após a otimização dos parâmetros experimentais, para os três
UMEs utilizados, alíquotas de pesticida no intervalo de concentração de $3,0 \times 10^{-6} \mathrm{~mol} \mathrm{~L}^{-1}$ a $1,66 \times 10^{-4} \mathrm{~mol} \mathrm{~L}^{-1}$, foram adicionadas à solução de $\mathrm{Na}_{2} \mathrm{SO}_{4} 0,1 \mathrm{~mol} \mathrm{~L}^{-1}$ e as intensidades de corrente de pico foram avaliadas após cada adição.

A Figura 3 apresenta os voltamogramas resultantes da adição de diferentes concentrações de paraquat analisados com UME de Pt com a inserção da curva de trabalho para o pico 1 e pico 2, onde é possível observar o aumento proporcional da corrente com o aumento da concentração do pesticida. Para os UMEs de Au e FC, um comportamento similar é observado.

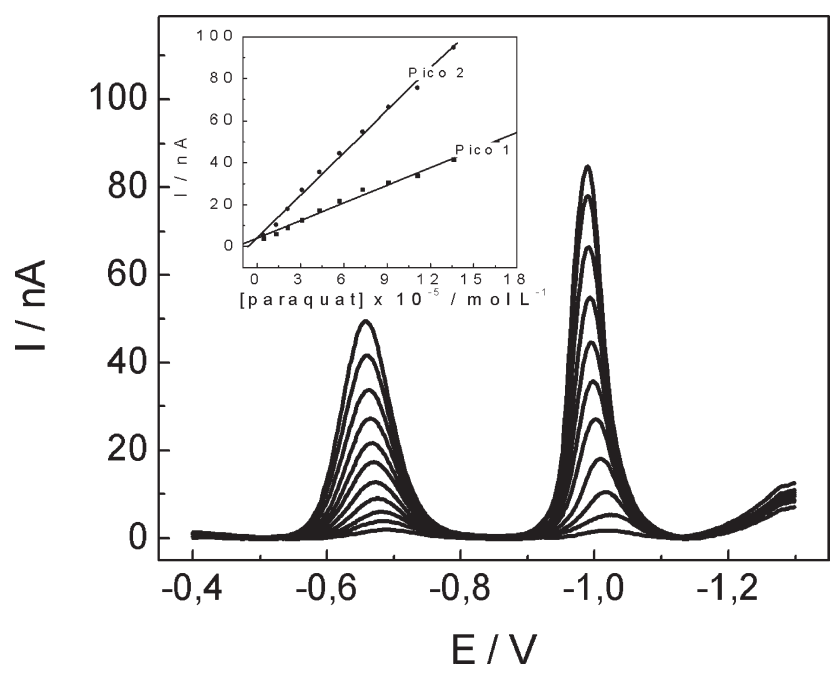

Figura 3. Voltamogramas de onda quadrada para o paraquat em UME de Pt, com $f=1000 \mathrm{~s}^{-1}, \Delta E_{p}=50 \mathrm{mV}, \Delta E_{s}=2 \mathrm{mV}$, meio de $\mathrm{Na}_{2} \mathrm{SO}_{4} 0,1 \mathrm{~mol} \mathrm{~L}^{-1}$ com concentrações de $3,0 \times 10^{-6}$ a $1,66 \times 10^{-4} \mathrm{~mol} \mathrm{~L}^{-1}$. Inserção: relação da corrente de pico com a concentração

A dependência linear das correntes de pico com a concentração de pesticida em solução, possibilitou a construção de curvas de trabalho para o paraquat sobre as três superfícies diferentes e o cálculo dos limites de detecção para os dois picos de redução, para posterior aplicação da metodologia na análise de águas naturais.

Os limites de detecção foram avaliados para os três UMEs utilizados utilizando-se a relação ${ }^{16}$ :

$L D=\frac{3 S_{b}}{s}$

onde $\mathrm{S}_{\mathrm{b}}$ é o desvio padrão da média de medidas do branco (média de 8 voltamogramas), tomadas no potencial equivalente àquele do pico da redução do paraquat e $s$ é a inclinação da reta de trabalho.

A Tabela 2 apresenta os resultados obtidos para as curvas de trabalho construídas a $1000 \mathrm{~s}^{-1}$ com a apresentação dos valores do desvio padrão das médias do branco, da inclinação da reta (s), do coeficiente de correlação (r) e do limite de detecção, considerandose os três UMEs utilizados considerando-se o pico 1 e a Tabela 3 apresenta os mesmos resultados considerando-se o pico 2 .

Tabela 2. Resultados obtidos para a detecção do paraquat em diferentes UMEs, a partir das curvas de trabalho obtidas do pico 1 . Freqüência de $1000 \mathrm{~s}^{-1}$

\begin{tabular}{lcccc}
\hline UME & $\mathrm{S}_{\mathrm{b}}(\mathrm{nA})$ & $\mathrm{s}\left(\mathrm{A} / \mathrm{mol} \mathrm{L}^{-1}\right)$ & $\mathrm{r}$ & $\mathrm{LD}\left(\mu \mathrm{g} \mathrm{L}^{-1}\right)$ \\
\hline $\mathrm{Pt}$ & 0,0168 & $2,810 \times 10^{-3}$ & 0,9952 & 3,9 \\
$\mathrm{Au}$ & 0,0442 & $4,796 \times 10^{-3}$ & 0,9995 & 6,2 \\
$\mathrm{FC}$ & 0,0521 & $1,701 \times 10^{-3}$ & 0,9972 & 20,39 \\
\hline
\end{tabular}


Tabela 3. Resultados obtidos para a detecção do paraquat em diferentes UMEs, a partir das curvas de trabalho obtidas do pico 2. Freqüência de $1000 \mathrm{~s}^{-1}$

\begin{tabular}{lcccc}
\hline UME & $\mathrm{S}_{\mathrm{b}}(\mathrm{nA})$ & $\mathrm{s}\left(\mathrm{A} / \mathrm{mol} \mathrm{L}^{-1}\right)$ & $\mathrm{r}$ & $\mathrm{LD}\left(\mu \mathrm{g} \mathrm{L}^{-1}\right)$ \\
\hline $\mathrm{Pt}$ & 0,1016 & $6,736 \times 10^{-3}$ & 0,9961 & 10,05 \\
$\mathrm{Au}$ & 0,1090 & $6,702 \times 10^{-3}$ & 0,9967 & 10,83 \\
$\mathrm{FC}$ & 0,1201 & $1,863 \times 10^{-3}$ & 0,9985 & 42,93 \\
\hline
\end{tabular}

Estes limites de detecção obtidos com a utilização de UMEs são bastante próximos de valores da literatura ${ }^{15,16}$ e muito apropriados para a determinação de paraquat em águas potáveis, onde o limite permitido pela US-EPA é de $0,1 \mathrm{ppm}$, ou seja, $100 \mathrm{ppb}^{17}$.

\section{CONCLUSÕES}

A utilização de ultramicroeletrodos no estudo do comportamento eletroanalítico do paraquat em água pura, de laboratório, mostrou algumas vantagens ainda não reportadas na literatura. A colaboração mais significativa, nesta área, é a possibilidade de se trabalhar com freqüência de aplicação de pulsos tão alta como $1000 \mathrm{~s}^{-1}$. Estes valores são praticamente impossíveis de se alcançar com eletrodos tradicionais devido, principalmente, às correntes capacitivas obtidas.

A grande importância de se trabalhar com frequiências tão elevadas está relacionada com: (a) diminuição dramática no tempo de análise, possibilitando uma economia significativa de tempo de equipamento, (b) melhora na sensibilidade do eletrodo, pois as correntes de pico são sempre diretamente proporcionais à freqüência.

Neste trabalho, os limites de detecção obtidos não são superiores àqueles reportados na literatura, para eletrodos convencionais com a utilização de frequiências mais baixas. Entretanto, ainda se pode otimizar significativamente o desempenho dos UMEs, por exemplo pela modificação de suas superfícies com Nafion ou outros agentes modificantes que melhorem a sua sensibilidade, ou pela utilização de conjuntos de UMEs de fibra de carbono, aumentando assim a dimensão da corrente medida no aparelho e, conseqüentemente, da sensibilidade da técnica.

\section{AGRADECIMENTOS}

Os autores agradecem ao CNPq e à FAPESP (Proc. Nos. 99/ 07891 e 00/03540-6) pelos auxílios concedidos.

\section{REFERÊNCIAS}

1. Klaassen, C. D.; Toxicology-the basic science of poisons (Casartt \& Doull's), 5th ed., International Ed.: New York, 1989.

2. Tampo, Y.; Tsukamoto, M.; Yonaha, M.; Free Radical Biol. Med. 1999, 27, 588.

3. Rai, M. K.; Das, J. V.; Gupta, V. K.; Talanta 1997, 45, 343.

4. Saad, B.; Ariffin, M.; Saleh, M. I.; Talanta 1998, 47, 1231.

5. Taguchi, V. Y.; Jenkins, S. W. D.; Crozier, P. W.; Wang, D. T.; J. Am. Soc. Mass Spectrom. 1998, 9, 830.

6. Walcarius, A.; Lamberts, L.; J. Electroanal. Chem. 1996, 406, 59.

7. Nuwer, M. J.; O’Dea, J. J.; Osteryoung, J.; Anal. Chim. Acta 1998, 362, 03.

8. Monk, P. M. S.; Turner, C.; Akhtar, S. P.; Electrochim. Acta 1999, 44, 4817.

9. Lu, T. H.; Sun, I, W.; Talanta 2000, 53, 443.

10. Osteryoung, J.; Osteryoung, R.; Anal. Chem. 1985, 57, 101A.

11. Souza, D.; Machado, S. A. S.; Avaca, L. A.; Quim. Nova 2003, 26, 81.

12. Osteryoung, J. Em Microelectrodes: Theory and Applications; Montenegro, M. I.; Queirós, M. A.; Dasbach, J., eds.; Applied Sciences: London, 1991.

13. Wightman, R. M.; Wipf, D. O.; Electroanalytical Chemistry ( a series of advances), Bard, A. J., ed.; Marcel Dekker: New York, 1984, vol. 14.

14. Zen, J. M.; Jeng, S. H.; Chen, H. J.; Anal. Chem. 1996, 68, 498.

15. Huebra, M. J. G.; Hernadéz, P.; Ballesteros, Y.; Hernadéz, L.; Talanta 2001, 54, 1077.

16. Fung, Y. S.; Mak, J. L. L.; Electrochim. Acta 1999, 44, 3855.

17. http://www.epa.gov/fedrgstr/EPA-PEST/1997/August/Day-29/p23094.htm, acessada em Junho 2002 